\title{
Controversed aspects regarding polymerization in dental technology
}

\author{
Irina Adriana BEURAN ${ }^{1}$, Mihai BURLIBASA ${ }^{1}$, Gabriela TANASE ${ }^{1}$, Augustin MIHAI $^{1}$, \\ Simion Gheorghe DUMITRU ${ }^{2}$, Radu COSTEA ${ }^{1}$, Iuliana BABIUC ${ }^{1}$, Viorel Stefan PERIEANU ${ }^{1}$, \\ Oana EFTENE ${ }^{1}$, Madalina Violeta PERIEANU ${ }^{1}$, Oana-Cella ANDREI ${ }^{1}$, Ruxandra STANESCU ${ }^{1}$, \\ Narcis MARCOV ${ }^{1}$, Elena-Cristina MARCOV ${ }^{1}$, Olivia POPOVICIU ${ }^{1}$ \\ 1 "Carol Davila" University of Medicine and Pharmacy, Bucharest, Romania \\ ${ }^{2}$ Dental practice, Brasov, Romania
}

\begin{abstract}
Introduction. Any new class of materials almost always brings with it a new processing technology, regardless of the field in which it is operated. If this technology is not fully respected, all the benefits and performance of the material can be compromised.

Purpose. In this material we tried not only a familiarization of the dental technicians, but rather their awareness regarding the polymerization processes, which intervene in various technologies for creating prosthetic restorations of any kind.

Material and method. For this study, 53 dental technicians, who have completed various forms of schooling, answered 10 questions related to the different forms of polymerization used in the dental technology.

Results and discussions. The answers obtained give us an image regarding the knowledge of the practitioners in this field, but also the way in which the theoretical knowledge is verified in practice by the daily experience of the respondents.

Conclusion. There is a variation in the accumulated knowledge, a variation that can be based both on the studies followed by each participant in the study and on how they use the materials discussed in this article in daily practice.
\end{abstract}

Keywords: dental technology, polymerization, dental materials

\section{INTRODUCTION}

The extraordinary development in the last 4 decades of polymers and composite materials used in dental medicine, in our case with reference to dental technology, would not have been possible without the introduction, launch and deepening of some mechanisms, but also of new devices, to carry out the polymerization.

Teaching students from undergraduate programs with a dental profile, especially those from dental medicine and dental technology, but also of dental practitioners and, in our case, dental technicians, regarding the initiation and learning the polymerization (curing) mechanisms of various materials with utility in the oral and dental care, including the dental technology, implies both the knowledge and as well as deepening some fields close to dentistry, namely the science of materials, with the characteristic notions of physics and chemistry. Many of the information 
related to the polymerization mechanisms of the materials used in the oral and dental care are taught both during post-secondary and/or university studies within the college of dental technology, but also later, during courses and/or post-university specializations. However, both students and participants in postgraduate programs, mostly refuse to assimilate the notions of chemistry and physics characteristic of these materials, arguing most often by the aridity of the details, by the lack of teaching method and the motivation of the readers (1-7).

\section{PURPOSE}

Starting from these aspects, before defining the actual purpose of this material, we will try a very brief explanation of some basic notions of the polymerization of the materials used in the oral and dental assistance, with particular reference to dental technology (8-10).

Thus, polymerization is that chemical reaction, whereby a compound, called monomer (denoted in our case with X), is transformed into polymer (in our case, macromolecular structure, $X_{n}$, where $n$ represents the degree of polymerization). In the dental technology we find materials that polymerize through several mechanisms, as it follows (1-3, 11-13):

1. Self-polymerization (self-curing) is a polymerization reaction, which takes place without the material requiring an external energy input, to initiate it. It is also called chemical polymerization. As advantages of this type of polymerization, we remind: it does not require polymerization equipment and it ensures a uniform polymerization, regardless of the material thickness. Among the disadvantages, the following should be mentioned: chromatic instability; incorporation of air during preparation; limited working time; continuous increase of the viscosity of the paste from the moment of preparation; increased toxicity; uncertain and relative homogenization.

2. Thermo-polymerization is a polymerization reaction, which requires an external caloric intake to initiate. The required temperature depends on the decomposition temperature of the initiator in free radicals. As processes, there is classical thermo-polymerization with slow and fast polymerization procedures, but also modern thermo-polymerization with humid heat (heat-curing by press process with humid heat; it differs from the classical thermo-polymerization in that the associated devices ensures besides a uniform heating, a pressure on model-resin-flask system, without the need for flat bench press) and heat-curing by press process with dry heat. As advantages of the thermo-polymerization should be mentioned: decrease in the percentage of residual monomer with the increase of the biocompatibility of the realized pieces, to which is added a safe and precise polymerization throughout the mass of the material. As disadvantages of thermo-polymerization, we mention only the most important ones: they require special equipment; requires a longer workload from the dental technician; the cost of prosthetic restorations obtained is higher than in the case of self-polymerization; there is a risk of air bubbles due to evaporation of the monomer when the thermal polymerization procedure is not respected. Also during thermo-polymerization, we discuss about obtaining polymeric injection-molded dentures which have multiple advantages: increased accuracy; superior mechanical resistance to breaking of the prosthetic restoration; time economy; better safety from the dental technician; fewer tweaks and corrections; absence of contact with the monomer; high polishing capacity; there is no risk of occlusion elevation.

Although, the thermo-polymerization comprises several processes, classic (with slow and/or fast polymerization procedure) and modern (press heat-curing polymerization with wet and dry heat), due to the limited space we have available, in this material we will only refer at the general mode, at the thermo-polymerization procedure, and we will not insist on all the existing variants, but that we have mentioned before.

3. Photo-polymerization (light curing) is the polymerization initiated by the external contribution of electromagnetic radiation, by irradiation with UV or visible (coherent and / or incoherent) radiation. The major advantage of the polymerization is that, the practitioner can trigger when he wants the polymerization (longer working time). Among the disadvantages, the following should be mentioned: it requires an additional equipment, which is quite expensive; the degree of polymerization is not always uniform throughout the mass of the material, but decreases between the irradiated surfaces to the depth.

The purpose of this report is both the familiarization of dental technicians with the basic notions regarding the polymerization processes, with the advantages and disadvantages of each one, but also the awareness of the same dental technicians about the importance of all the maneuvers of obtaining prosthetic restorations of any type, fixed and mobile, involving polymerization maneuvers of different materials, with the specific equipment and instrumentation of these extremely complex and laborious processes (4-13). 


\section{MATERIAL AND METHOD}

The training of those involved in the dental practice, whether we are talking about dental doctors and/or dental technicians, implies a very good knowledge and in-depth knowledge of the characteristics of the materials that they use. But, this requires from the specialized personnel, and solid knowledge in the fields related to dentistry, namely material science, with general knowledge of physics and chemistry, without which these basics cannot be understood and/or learned.

This study is mainly addressed to the dental staff who work in the technical department, so dental technicians and pursues 2 interesting objectives, namely (8-13):

- acquainting with the basic notions regarding the polymerization processes, with the advantages and disadvantages of each;

- raising the awareness of dental technicians on the importance of all the maneuvers for obtaining prosthetic restorations of any kind, fixed or mobile, which involve polymerization maneuvers of different materials, with the equipment and in-

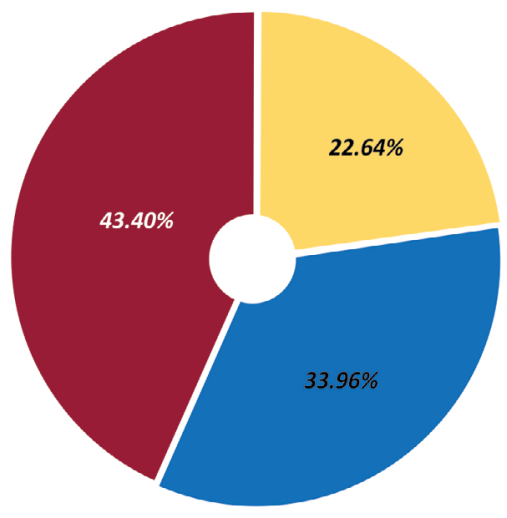

$\square$ post-high school graduates $\square$ college graduates $\square$ university graduates

FIGURE 1. Distribution of the subjects included in the study according to the studies they followed strumentation specific to these extremely complex and laborious processes.

The method selected for this study was the questionnaire. It was made up of a number of 10 questions and was applied to 53 subjects, dental technicians, 12 post-high school education graduates (representing $22.64 \%$ ), 18 college graduates (representing $33.96 \%$ ) and 23 graduates of university studies (representing 43.40\%) (Fig. 1). Subjects were aged between 27 to 63 years, and the study was conducted subject to anonymity. The gender distribution of the 53 subjects included in the study was as follows: 35 subjects (representing $66.04 \%$ ) were female, while 18 subjects (representing $33.96 \%$ ) were male (Fig. 2).

The questionnaire applied to the 53 subjects was the following:

1. Self-polymerization (self-curing) is? a. A polymerization reaction, which takes place without the material requiring an external energy input, to initiate it; $\boldsymbol{b}$. A reaction that automatically requires a heat source; $c$. Also called chemical polymerization or chemo-polymerization.

\section{Correct answers: $\boldsymbol{a}$. c.}

2. Of the advantages of self-polymerization, the most important are? a. It does not require polymerization equipment; $\boldsymbol{b}$. It ensures a uniform polymerization, regardless of the material thickness; $c$. It is the material of choice in the plating of metallic-polymer fixed prosthetic restorations.

\section{Correct answers: $\boldsymbol{a}, \boldsymbol{b}$.}

3. The disadvantages of self-polymerization can be? a. Incorporation of air during preparation; b. Limited working time and continuous increase of the viscosity of the paste from the moment of preparation; $\boldsymbol{c}$. Very low toxicity; $\boldsymbol{d}$. Uncertain and relative homogenization; e. An exceptional chromatic.

Correct answers: $a, b, d$.

4. The thermo-polymerization methods are? a. Classic thermo-polymerization with slow polymerization regime; $\boldsymbol{b}$. Classic thermo-polymerization

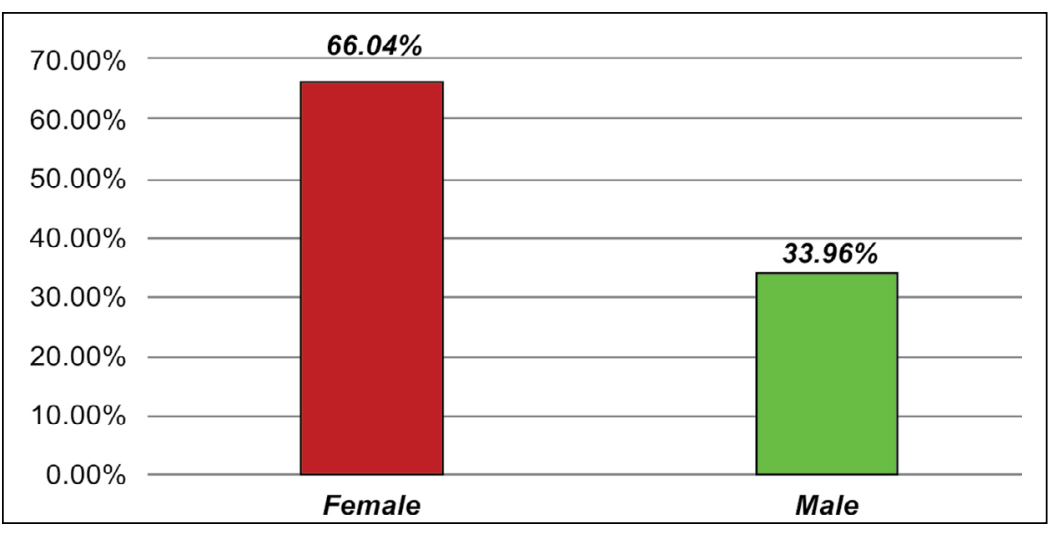

FIGURE 2. Gender distribution of the respondents 
with rapid polymerization regime; c. Modern thermo-polymerization with humid heat (press heat-curing polymerization with humid heat); $\boldsymbol{d}$. Thermo-polymerization with dry heat; $\boldsymbol{e}$. Thermo-polymerization with photochemical initiation.

\section{Correct answers: $\boldsymbol{a}, \boldsymbol{b}, \boldsymbol{c}, \boldsymbol{d}$.}

5. Which of the following are advantages of thermo-polymerization? a. Decreasing the percentage of residual monomer with increasing the biocompatibility of the pieces made; $\boldsymbol{b}$. The polymerization time is very short, 3-5 minutes; c. A safe and precise polymerization throughout the mass of the material.

\section{Correct answers: $\boldsymbol{a}, \boldsymbol{c}$.}

6. The most important disadvantages of thermo-polymerization are? a. Requires special equipment; $\boldsymbol{b}$. It requires a longer workload from the dental technician; $c$. There is a risk of air bubbles occurring when the thermal polymerization regime is not respected; $\boldsymbol{d}$. Initiation is done with the help of ultraviolet light;

\section{Correct answers: $\boldsymbol{a}, \boldsymbol{b}, \boldsymbol{c}$.}

7. Among the advantages of polymeric dentures obtained by injection method are? a. Superior mechanical resistance to rupture of prosthetic restoration and time savings; b. Superior safety and precision from the dental technician; c. Less tweaks and corrections, but also the absence of contact with the monomer; $\boldsymbol{d}$. Higher polishing capacity; $\boldsymbol{e}$. There is no risk of occlusion elevation; $f$. Higher costs compared to CAD / CAM technology.

\section{Correct answers: $a, b, c, d$.}

8. Photo-polymerization (light curing) represents the polymerization initiated by? $\boldsymbol{a}$. The external contribution of electromagnetic radiation; $\boldsymbol{b}$. By irradiation with UV or visible radiation (coherent and / or incoherent); c. By large caloric intake.

\section{Correct answers: $\boldsymbol{a}, \boldsymbol{b}$.}

9. The advantages of the photo-polymerization (light curing) regime are? $\boldsymbol{a}$. The practitioner can trigger when he wants the polymerization (longer working time); b. Requires additional equipment, quite expensive; $\boldsymbol{c}$. The degree of polymerization is not always uniform throughout the mass of the material, but decreases between the irradiated surfaces to the depth.

\section{Correct answer: $\boldsymbol{a}$.}

10. Using both the theoretical information presented and learned in this material, but also the daily experience from the practical activity, which of the polymerization methods do you consider to be the most efficient and cost-effective of the technologies for creating prosthetic restorations? $\boldsymbol{a}$. Self-polymerization (self-curing); b. Photo-polymerization (light curing); c. Thermo-polymerization, regardless of the variant used? (Only one answer)

\section{RESULTS AND DISCUSSIONS}

We present the results obtained by applying the questionnaire.

For the first question about self-polymerization 37 respondents (representing 69.81\%) have answered correctly (without external energy input, chemical polymerization), while only 16 respondents (representing 30.19\%) have answered incorrectly including in their answers the necessity of a heat source (Fig. 3).

Regarding the advantages of self-polymerization, 38 dental technicians included in the study (representing $71.70 \%$ ) have answered correctly, it does do not require special equipment, respectively they ensure a uniform polymerization. Only 15 technicians included besides the correct answers, the material of choice option, which is otherwise a wrong answer (Fig. 4).

For the third question, all the respondents included in the study answered correctly: namely, air intake during preparation, limited working time and uncertain homogenization.

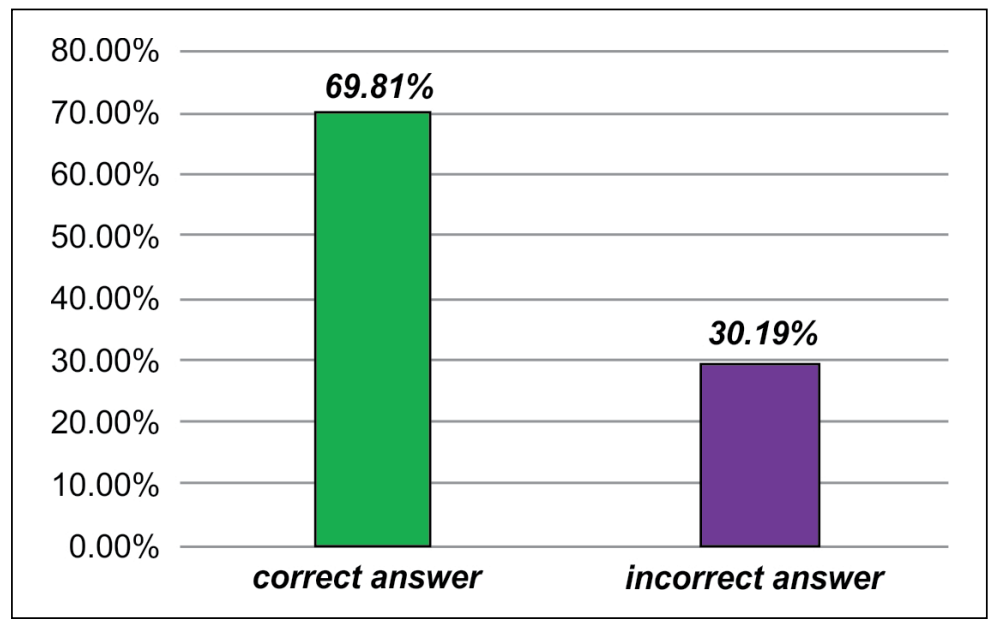

FIGURE 3. Assessment of knowledge about self-polymerization 


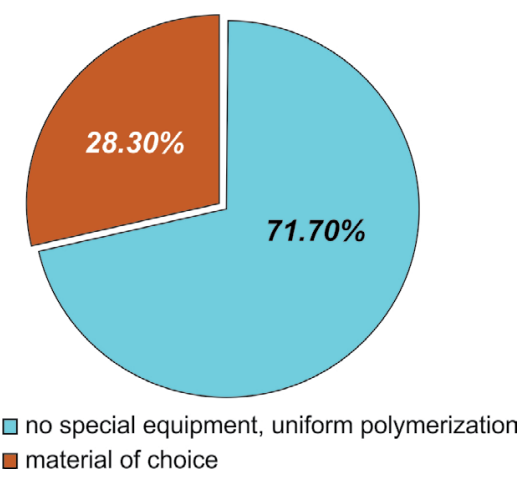

FIGURE 4. The advantages of self-polymerization

Regarding the processes of thermo-polymerization most of the subjects (50 - representing 94.34\%) have answered correctly: classic with slow regime, classic with fast regime, with humid heat and dry heat. Only 3 subjects (representing $5.66 \%$ ) included in the answers the wrong variant of photo-chemical initiation (Fig. 5).

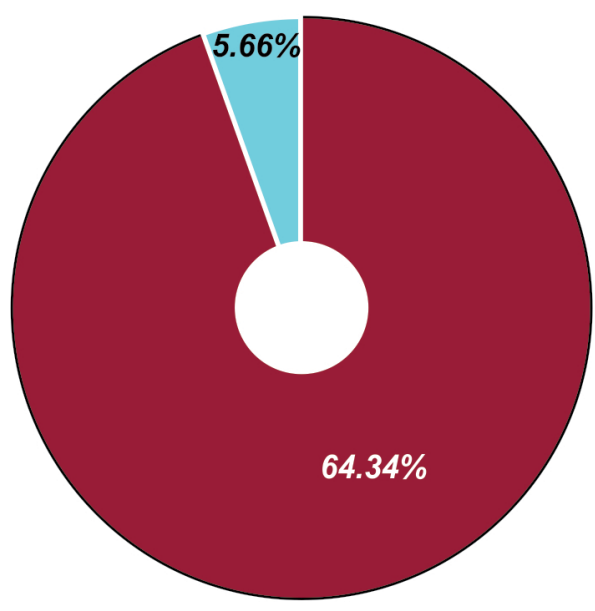

Correct answer $\square$ Wrong answer

FIGURE 5. Types of thermo-polymerization

42 of the technicians (representing 79.25\%) are well aware of the advantages of thermo-polymerization (decrease of monomer percentage and polymerization throughout the mass of the material). Only 11 technicians (representing $20.75 \%$ ) responded incorrectly, including the very fast polymerization time (Fig. 6).

The disadvantages of thermo-polymerization are well known by the technicians included in the study, all answering correctly the sixth question.

Polymer dentures obtained by injection technique, although it is newer technology, are well known among the technicians included in the study, 45 of them (representing $84.90 \%$ ) have answered correctly (superior mechanical strength, safety and precision, less retouching and no risk of elevation occlusion), while 8 technicians (representing $15.10 \%$ ) have answered incorrectly (Fig. 7).

Both in terms of initiating light curing and the advantages of the light curing regime, all respondents provided the correct answers.

The last question tries to reach a conclusion by combining the theoretical part with the practical part, in the desire to find out from the practitioners which of the polymerization methods seems to be the most efficient and cost effective, as follows:

- 40 technicians (representing 75.47\%) have responded thermo-polymerization regardless of the variant used;

- 12 technicians (representing 22.64\%) have answered the light curing;

- 1 technician (representing 1.89\%) have answered the self-polymerization (Fig. 8).

\section{CONCLUSIONS}

After studying the answers to the 10 questions, we can conclude some important aspects.

The resistance of the prosthetic restorations obtained by thermo-polymerization regimes, regardless of the variant, is significantly higher compared to the prosthetic restorations obtained by self-curing and light curing regime, which is why most dental technicians prefer the technologies and materials with thermo-polymerization regime.

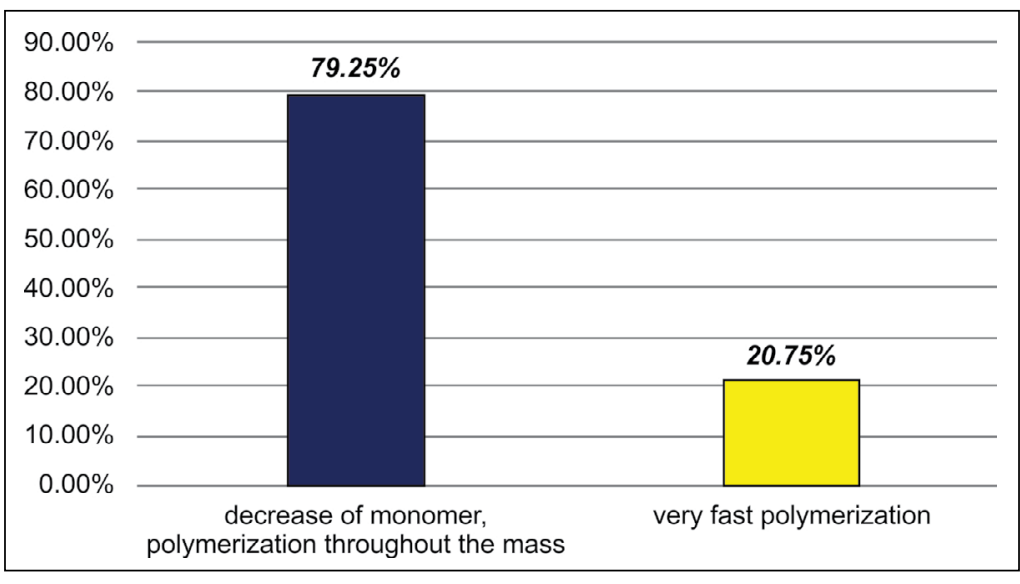

FIGURE 6. The advantages of thermo-polymerization 


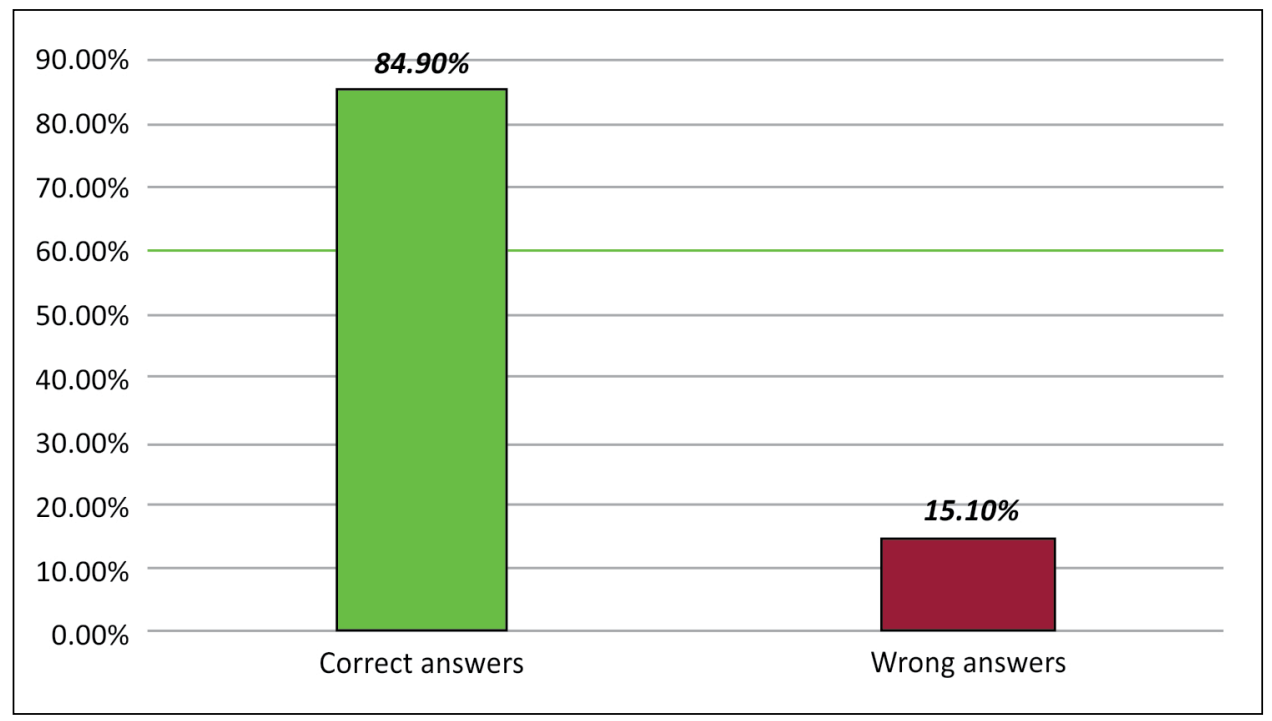

FIGURE 7. The advantages of the polymeric dentures obtained by injection technique

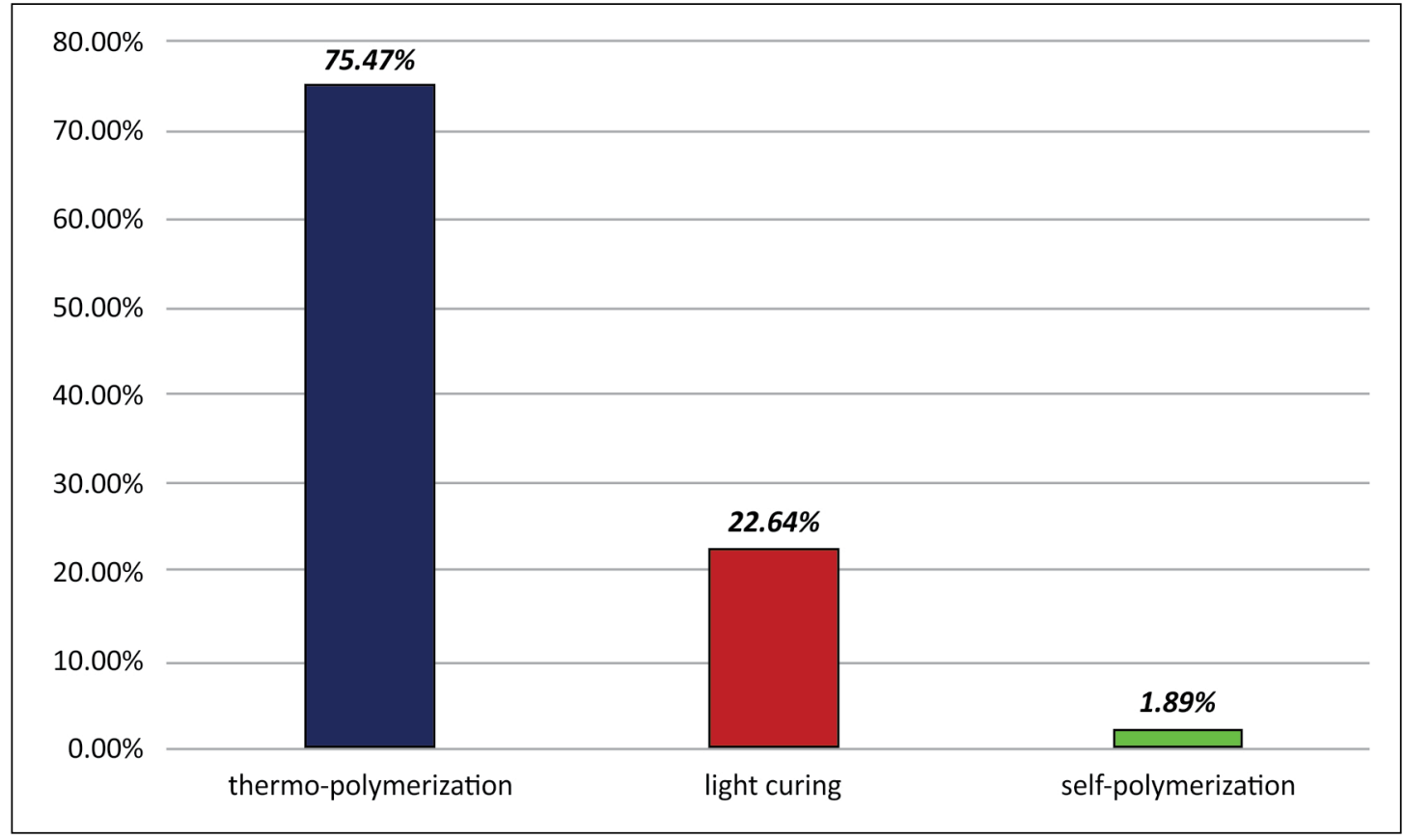

FIGURE 8. Evaluation of the polymerization methods by the technicians participating in the study

The cost of prosthetic restorations obtained by thermo-polymerization is higher, compared to those obtained by self-polymerization.

The working time of making prosthetic restorations by thermo-polymerization techniques is longer, than in the case of the other 2 processes, namely self-curing and light curing.

The toxicity of the self-curing materials is much higher, compared to the toxicity of the thermo-polymerization and light curing materials for both the user and the beneficiary, which is why the most acceptable restorations are the prosthetic restorations obtained by means of thermo-polymerization, regardless of variant.
Although the equipment's for the thermo-polymerization curing process, regardless of the variant used, are definitely much more expensive and laborious than in the case of the self-polymerization process, but not comparing with light curing, almost all dental technicians prefer the technology of thermo-polymerization in the majority of prosthetic restorations manufacturing, at the expense of self-curing and light curing.

The degree of understanding of the knowledge related to polymerization for the subjects included in the study varies from person to person, depending on the studies carried out by each dental technician (post-high school, college, university stud- 
ies), the degree of basic knowledge (physical and chemistry) that they have accumulated during the pre-technical dental period (gymnasium and high school), but also by the way they use in their daily practice materials with these polymerization regimes. Of course, we should discuss the major differences in training between post-high school studies, compared to college and especially university studies, but this was not the topic of the present study.

\section{Acknowledgement}

In this article, all the authors have equal contribution with the first author.

Conflict of interest: none declared

Financial support: none declared

\section{REFERENCES}

1. Romînu M, Bratu D, Lakatos S, Florița Z. Polimerizarea în stomatologie. Editura Brumar, Timișoara, 2000.

2. Bratu $D$ și colab. Materiale dentare în laboratorul de tehnică dentară, Vol. III. Editura Helicon, Timișoara, 1994.

3. Pătrașcu I și colab. Materiale dentare, Editura Horanda Press, București, 2002.

4. Cristache $\mathrm{CM}$, Burlibașa $\mathrm{M}$, Cristache $\mathrm{G}$ et al. Zirconia and its biomedical applications. Metalurgia International. 2011; Vol. XVI, No. 7, p. 18-23.

5. Burlibașa M, Muntianu LAS, Tănase $G$ et al. Study on microbial contamination of biomaterials in medical practice. Metalurgia International. 2010; Vol. XV, Spec. Iss. 2, p. 163-166.

6. Burlibașa M, Cristache CM, Georgescu SR et al. Toxicity of titan and nikel-cobaltchromium alloys. Metalurgia International. 2009,14 , p. $20-22$.
7. Burlibașa L, Gavrila L. Developmental epigenetics: roles in embryonic development, in Nutrition in Epigenetics (eds. Niculescu M.D., Haggarty P.), 2011, Ch. 6, p. 105-126, Willey-Blackwell Publishing.

8. Cristache $\mathrm{CM}$, lonescu $\mathrm{C}$, Cristache $\mathrm{G}$ et al. A 5-year prospective randomised clinical trial on the efficiency of two different attachment systems as retention for implant-supported mandibular overdenture. Radiographic assesment, cost analysis and final evaluation of treatment's success. Metalurgia International. 2009; Vol. XIV, Spec. Iss. No. 16, p. 27-34.

9. Cristache $\mathrm{CM}$, Ionescu $\mathrm{C}$, Burlibașa $\mathrm{M}$ et al. Rettentive anchors versus magnets as attachment systems for mandibular overdenture. A 5 year prospective randomised clinical study. Metalurgia International. 2009; Vol. XIV, Spec. Iss. No. 16 , p. 59-64.
10. Tănase $G$, Burlibașa $M$, Muntianu L et al. Testing the antibacterial potential of biomaterials in medical practice. Metalurgia International. 2010; Vol. XV, Spec. Issue No. 2, p. 160-162.

11. Burlibașa $M$, Cernușcă-Mițariu M, Burcea $\mathrm{CC}$ et al. Halogen compounds - theoretical, physiological and practical aspects regarding the decontamination, disinfection and sterilisation of intsruments and biomaterials in dental medicine practice. Metalurgia International. 2013; Vol. XVIII, Spec. Issue No. 3, p. 54-57.

12. Burlibasa L, Domnariu C. Epigenetic landscape of human diseases. Acta Medica Transilvanica. 2018; 23(2); 33-37.

13. Bodnar DC, Burlibașa L, Varlan C et al. Mercury, biocompatibility and its impact on environment. Metalurgia International. 2009; 14, 95-100. 